\title{
echinus, required for interommatidial cell sorting and cell death in the Drosophila pupal retina, encodes a protein with homology to ubiquitin-specific proteases

\author{
Jeffrey M Copeland ${ }^{1}$, Ian Bosdet ${ }^{2}$, J Douglas Freeman², Ming Guo ${ }^{3}$, \\ Sharon M Gorski ${ }^{2}$ and Bruce A Hay*1
}

Address: ${ }^{1}$ Division of Biology, MC 156-29, California Institute of Technology, Pasadena, CA 91125, USA, ${ }^{2}$ Genome Sciences Centre, British Columbia Cancer Agency. Vancouver, British Columbia V5Z 1L3, Canada and ${ }^{3}$ Department of Neurology, Brain Research Institute, The David Geffen School of Medicine at UCLA, Los Angeles, CA 90095, USA

Email: Jeffrey M Copeland - copelanj@its.caltech.edu; Ian Bosdet - ibosdet@bcgsc.ca; J Douglas Freeman - dfreeman@bcgsc.ca; Ming Guo - mingfly@ucla.edu; Sharon M Gorski - sgorski@bcgsc.ca; Bruce A Hay* - haybruce@caltech.edu

* Corresponding author

Published: 5 July 2007

BMC Developmental Biology 2007, 7:82 doi:10.1186/147|-2/3X-7-82

This article is available from: http://www.biomedcentral.com/I47I-2/3X/7/82

(C) 2007 Copeland et al; licensee BioMed Central Ltd.

This is an Open Access article distributed under the terms of the Creative Commons Attribution License (http://creativecommons.org/licenses/by/2.0), which permits unrestricted use, distribution, and reproduction in any medium, provided the original work is properly cited.
Received: 18 January 2007

Accepted: 5 July 2007

\begin{abstract}
Background: Programmed cell death is used to remove excess cells between ommatidia in the Drosophila pupal retina. This death is required to establish the crystalline, hexagonal packing of ommatidia that characterizes the adult fly eye. In previously described echinus mutants, interommatidial cell sorting, which precedes cell death, occurred relatively normally. Interommatidial cell death was partially suppressed, resulting in adult eyes that contained excess pigment cells, and in which ommatidia were mildly disordered. These results have suggested that echinus functions in the pupal retina primarily to promote interommatidial cell death.

Results: We generated a number of new echinus alleles, some likely null mutants. Analysis of these alleles provides evidence that echinus has roles in cell sorting as well as cell death. echinus encodes a protein with homology to ubiquitin-specific proteases. These proteins cleave ubiquitin-conjugated proteins at the ubiquitin C-terminus. The echinus locus encodes multiple splice forms, including two proteins that lack residues thought to be critical for deubiquitination activity. Surprisingly, ubiquitous expression in the eye of versions of Echinus that lack residues critical for ubiquitin specific protease activity, as well as a version predicted to be functional, rescue the echinus loss-offunction phenotype. Finally, genetic interactions were not detected between echinus loss and gainof-function and a number of known apoptotic regulators. These include Notch, EGFR, the caspases Dronc, Drice, Dcp-I, Dream, the caspase activators, Rpr, Hid, and Grim, the caspase inhibitor DIAPI, and Lozenge or Klumpfuss.

Conclusion: The echinus locus encodes multiple splice forms of a protein with homology to ubiquitin-specific proteases, but protease activity is unlikely to be required for echinus function, at least when echinus is overexpressed. Characterization of likely echinus null alleles and genetic interactions suggests that echinus acts at a novel point(s) to regulate interommatidial cell sorting and/or cell death in the fly eye.
\end{abstract}




\section{Background}

The adult Drosophila eye consists of 750-800 individual unit eyes, known as ommatidia, which are arranged in a hexagonal lattice. Each ommatidium consists of 8 photoreceptors, 4 lens-secreting cone cells and 2 primary pigment cells. Ommatidia are separated from each other by secondary and tertiary $\left(2^{\circ}\right.$ and $\left.3^{\circ}\right)$ pigment cells, and by sensory bristles. Each of these cell types occupies a stereotypic position within the lattice. Pattern formation in the eye is initiated in the 3rd larval instar as a wave of morphogenesis sweeps across the epithelial cell layer in the eye imaginal disc. First, eight photoreceptor cells and four lens-secreting cone cells are specified through sequential inductive interactions. During early pupal stages, cone cells come to cover the photoreceptors. They also recruit two primary pigment cells, which surround the cone cells. Cells that have not been specified at this stage form the interommatidial cell (IOC) lattice, which will ultimately be composed of secondary pigment cells, tertiary pigment cells, and bristles. These cells initially appear undifferentiated and unpatterned, with several layers of IOCs often separating neighboring ommatidia. Reorganization begins with presumptive lattice cells maximizing their contacts with primary pigment cells rather than with other lattice cells. This results in each lattice cell being connecting to at least two primary pigment cells, and with each ommatidia being separated by a single layer of lattice cells, arranged in an end-to-end chain. About two-thirds of these cells will go on to develop as secondary pigment cells, each of which makes up one face of the ommatidial hexagon, or tertiary pigment cells, which make up alternative vertices, with bristle groups making up the other vertices. The remainder of the IOCs are eliminated by apoptotic cell death $[1,2]$.

Much cell death in Drosophila takes the form of apoptosis [3]. Caspase proteases are the central executioners of apoptotic cell death [4]. Dronc is required for many cell deaths in the fly [5-8], including those of the IOCs [9]. Once activated through interactions with the adaptor Ark, Dronc cleaves and activates effector caspases such as Drice and Dcp-1 that are thought to bring about cell death $[5,6]$. Drice is activated during the stages in which IOC death occurs [10], and Drice mutants lack some, but not all, IOC death, highlighting the importance of this protease $[11,12]$. DIAP1 is a cell death inhibitor that suppresses the activity of Dronc and caspases activated by Dronc through several different mechanisms [5,6,13-19]. Reaper (Rpr) [20], Head involution defective (Hid) [21], Grim [22], Sickle [23-25], and Jafrac2 [26], known collectively as the RHG proteins after their founding members Rpr, Hid and Grim, bind to DIAP1 through a short-N-terminal motif and disrupt DIAP1-caspase interactions through several mechanisms, each of which has the effect of unleashing a cascade of apoptosis-inducing caspase activity. Flies that lack Hid show defects in Drice activation and IOC cell death $[10,27]$, while mutants for the other proteins are not available. Together these observations suggest that IOC death is driven, at least in part, by Hid-dependent inhibition of DIAP1, which facilitates activation of Dronc and Drice (Fig. 4 schematic).

Ubiquitination, and thus presumably deubiquitination, plays several important roles in the regulation of this cell death pathway. DIAP1 is an E3 ubiquitin ligase [28-32] that can ubiquitinate and inactivate Dronc $[15,16]$. DIAP1 can also promote the ubiquitination and degradation of other pro-apoptotic proteins that it binds such as Reaper [33]. DIAP1 also ubiquitinates itself [28-32,34] and DIAP1 ubiquitination can be stimulated by the RHG proteins [28-32,34]. Many components of the ubiquitin pathway have been identified as regulators of RHG-mediated cell death. Examples include the ubiquitin activating enzyme (uba1), two components of an SCF-type E3 ubiquitin ligase (skpA and a novel F-box gene, morgue) and the deubiquitinating enzyme fat facets [29,31,32]. However, the points at which these proteins work to regulate death are largely unknown.

As noted above, the IOCs are initially arranged in double or triple rows between ommatidia in the pupal eye. IOCs then rearrange in an end-to end configuration to form a single layer or row of cells separating primary pigment cells of different ommatidia. Cell death only occurs after this rearrangement, or sorting, is complete. A key player in this process is the immunoglobulin family protein Roughest (Rst). In the absence of roughest the IOCs remain stacked side-by-side in multiple rows and IOC death does not occur [2]. Rst is localized in IOCs to the border between IOCs and primaries [35], in a process that requires DE-cadherin and Notch [36-38]. Recent observations suggest that Rst promotes sorting by physically interacting with Hibris, another immunoglobulin family membrane protein expressed in primary pigment cells that is also required for IOC sorting and death [37].

The EGF receptor pathway provides important anti-apoptotic signals to IOCs. Loss of EGFR signaling in the pupal eye results in fewer IOCs [39], while activation of EGFR or Ras promotes IOC survival [40]. EGFR activation promotes IOC survival at least in part by negatively regulating hid levels and pro-apoptotic activity $[10,27,41]$. Pro-survival signaling through the EGFR is antagonized by Notch-mediated signals (probably between IOCs), which are required to remove excess IOCs $[10,40]$. The amount of contact an IOC has with primary pigment cells (which produce EGFR-dependent survival signals) as opposed to other IOCs (which produce Notch-mediated signals that antagonise the EGFR pathway) is likely to be an important part of the calculus that determines IOC fate. Ubiquitina- 
tion plays important roles in regulating both signaling pathways. The EGFR is monoubiquitinated following ligand binding, and this promotes receptor endocytosis and degradation, thus attenuating signaling [42]. In the Notch pathway, monoubiquitination of both ligands and Notch by multiple E3 ligases is associated with endocytic events that promote signaling. Ubiquitination of Notch by other E3 ligases is associated with internalization and lysosomal degradation [43].

Other proteins that regulate IOC survival have been identified. The Runx DNA-binding protein Lozenge is required for IOC death [44-46]. Lozenge pro-apoptotic activity is mediated by its ability to induce the expression of Argos, a secreted inhibitor of EGFR activation in cone cells, and $2^{\circ}$ and $3^{\circ}$ cells [44]. Lozenge also activates expression of Klumpfuss, a transcription factor with similarity to the Wilm's Tumor suppressor, in $2^{\circ}$ and $3^{\circ}$ cells [44]. Klumpfuss function is required for normal levels of IOC death, and genetic evidence suggests that it antagonizes EGFR signaling downstream of receptor activation [47].

echinus (ec), defined by a single allele, $e c^{1}$, was identified by Calvin Bridges in 1918 as a X-chromosome-linked, recessive, rough eye mutant (as described in [48]). More recently, Wolff and Ready showed that $e c^{1}$ flies had decreased levels of IOC death, much like rst mutants. However, while IOC sorting failed to occur in rst animals, sorting was largely (though not completely) intact in $e c^{1}$ flies $[2,49]$. Expression of the baculovirus caspase inhibitor $\mathrm{p} 35$ also prevents death but not sorting, giving rise to a pupal retinal phenotype with many similarities to (though not identical to) that observed in $e c^{1}$ flies [50]. Together these observations have suggested that $e c$ acts primarily subsequent to sorting, perhaps regulating cell death directly [49-51]. To understand the role echinus plays in bringing about IOC death we generated a number of new echinus alleles and re-examined phenotypes associated with $e c^{1}$ following extensive outcrossing to remove modifiers. Multiple alleles, including putative null alleles, show defects in cell sorting as well as cell death. We cloned the echinus locus and found that it encodes multiple isoforms of a protein with homology to the ubiquitin specific protease (USP) family of proteases. Somewhat unexpectedly, versions of Echinus that lack residues thought to be important for USP catalytic activity can rescue the echinus loss-of-function sorting and cell death phenotypes. We were unable to detect significant interactions between loss- and gain-of-function echinus alleles and a number of known or suspected regulators of IOC death. echinus may primarily regulate cell sorting, with loss of death being only a consequence of an earlier defect in this process. Alternatively, echinus may regulate cell sorting and cell death, with regulation of death occurring at a novel point, perhaps through mechanisms that are independent of USP activity.

\section{Results \\ CG2904 encodes echinus, which is expressed at low levels ubiquitously in the pupal retina}

As a first step to cloning the echinus locus we used both EMS mutagenesis and $P$ element excision to generate new echinus alleles. We identified an X chromosome-linked $\mathrm{P}$

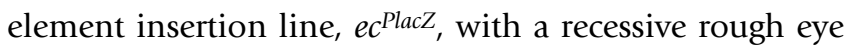
phenotype that failed to complement $e c^{1}$ (Fig. 1A). We generated a number of excisions of this element. The rough eye phenotype was reverted in some of these, indicating that the P element insertion was responsible for the echinus phenotype. The ec $c^{\text {PlacZ }}$ transposon is located between CG2901 and CG2904, suggesting one or the other of these genes as good candidates to encode echinus.

Ommatidia from wildtype flies are arranged in a regular hexagonal pattern (Fig. 2A), and extra IOCs are not observed in pupal eyes (Fig. 2G; Table 1). In contrast, adult eyes of $e c^{1}$ flies are rough (Fig. 2B), and extra IOCs are present in the pupal retinas (Fig. 2H). To determine if CG2904 encodes echinus we generated flies expressing dsRNA corresponding to CG2904 in order to trigger RNAi-dependent knockdown of CG2904 expression, under the control of the eye-specific-GMR promoter (GMR-CG2904-RNAi). Consistent with this hypothesis, GMR-CG2904-RNAi flies had a rough eye phenotype (Fig. 2C) and extra IOCs (Fig. 2I). To create deletion alleles of CG2904 we generated excisions from a nearby P element insertion line, $E P(X) 1343$, that is wildtype with respect to echinus (Fig. 1A). Multiple excision lines were identified that had rough eyes as homozygotes. Each of these failed to complement $e c^{1}$ or $e c^{\text {PlacZ }}$. Breakpoints for four of these were determined, and each was found to delete sequences within the CG2904 transcript. We have focused our analysis on one of these, designated $e c^{E P \Delta 4}$ (Fig. 1A; Fig 1D, J). Pupal eyes from $e c^{E P \Delta 4}$ also showed extra IOCs (Fig. 2J). We found that the CG2904 gene was also disrupted by the breakpoints of an excision allele generated from an alternate P element insertion $\left(e c^{\Delta 9}\right.$; Figure $1 \mathrm{H}$. Kramer, unpublished). This mutant also had rough eyes and extra IOCs (data not shown). More recently, a number of new P element insertion lines in the surrounding genomic region have been identified [52]. Several have a rough eye phenotype that fails to complement $e c^{1}$, and each of these is located near CG2904 (Figure 1A). We also identified several new EMS alleles of echinus (see methods for details). We sequenced CG2904 coding sequences from one of these and identified an E125-Stop change in $e c^{56}$ (Figure 1A). We also identified a stop mutation (L792-Stop in splice form 1) in the EMS-derived $e c^{3 c 3}$ allele [53]. Finally, we sequenced CG2904 coding and nearby regions from the original $e c^{1}$ stock. Two significant alterations were 


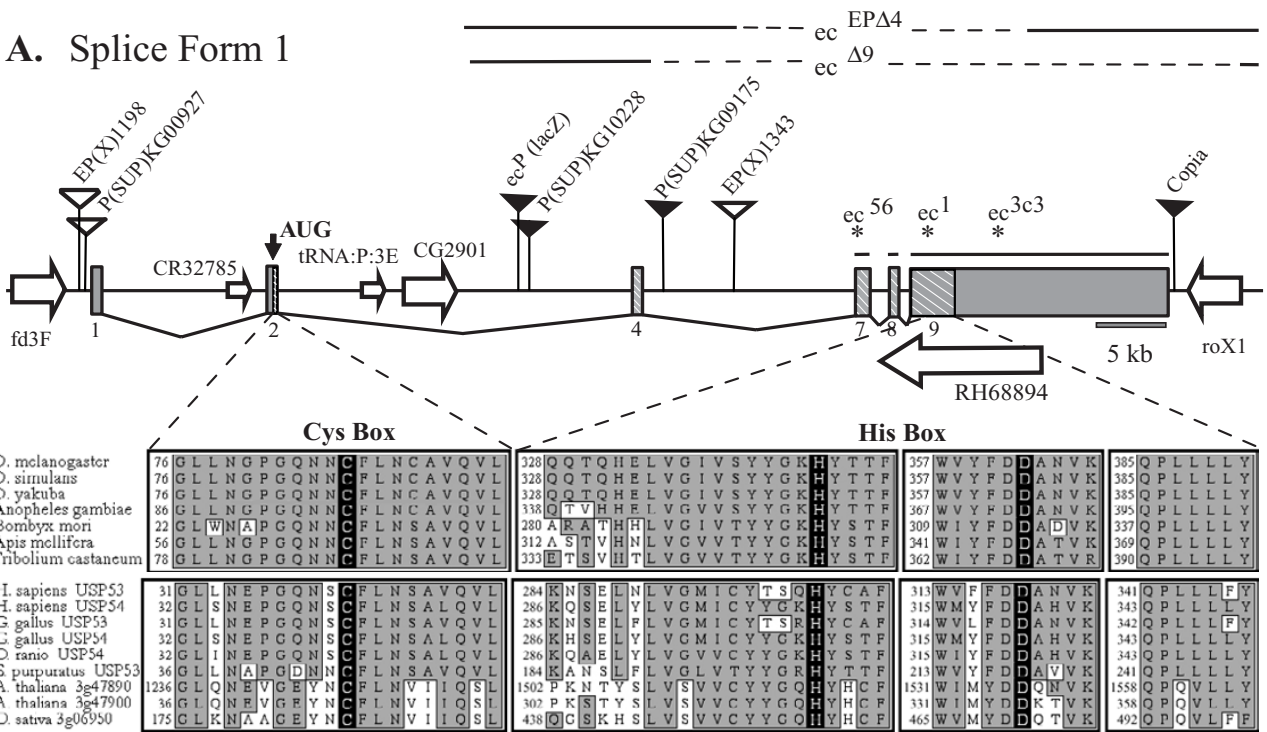

B. Splice Form 2
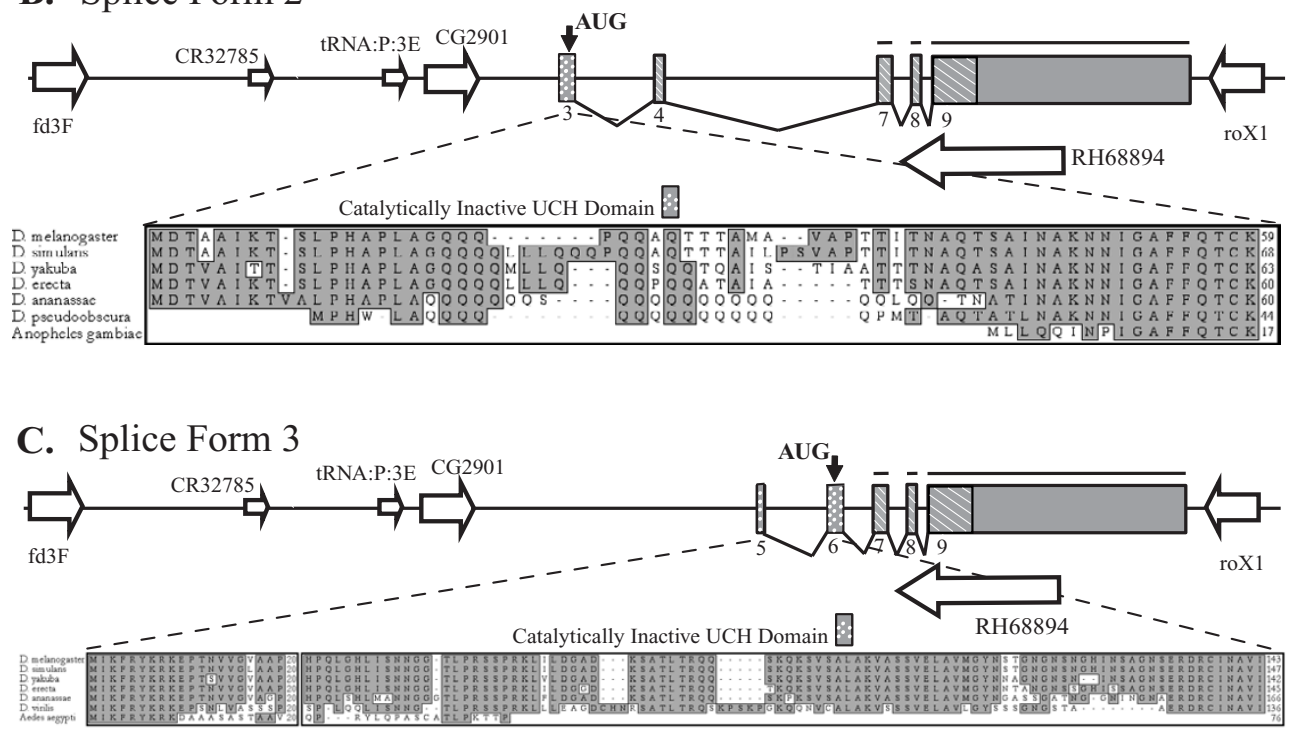

Figure I

echinus gene structure, mutants and surrounding genomic region. Echinus (CG2904) exons are indicated by shaded boxes. Exons are numbered sequentially with respect to the $5^{\prime}$ end of the gene. The shaded boxes with diagonal lines represent the conserved USP domain. Three different splice versions identified through cDNA sequencing are illustrated in panels A-C. Exons common to all three splice versions are noted with lines above numbered exons 7,8, and 9. Nearby genes are indicated (open arrows), as are Flybase annotated $\mathrm{P}$ element insertions, and ec PlacZ. Open triangles indicate $\mathrm{P}$ element insertions that are wildtype with respect to echinus, while filled triangles indicate $P$ element insertion lines with rough eyes that fail to complement echinus. The location of EMS-induced point mutations in echinus, ec ${ }^{56}$ and $e c^{3 c 3}$, and mutations identified in ec (a point mutation and a Copia insertion) are indicated by asterisks. The locations of the breakpoints for the echinus deletion alleles ec ${ }^{E P \Delta 4}$ and $e^{\Delta}{ }^{\Delta}$ are indicated by dotted lines at the top. (A) ec-SFI encodes a version of echinus that contains Cys and His box residues important for USP catalysis, as noted by the highlighted residues. These and surrounding sequences are highly conserved in predicted echinus homologs in other insect species. Highly related sequences are also found in a number of other species. (B) The ec-SF2 transcript initiates at a downstream exon, which contains an initial methionine and coding sequences that lack a Cys box. The 3' exon containing His box sequences (exon 9) is still present. (C) The ec-SF3 transcript initiates at a distinct position further $3^{\prime}$, and also contains His box sequences, but lacks Cys box sequences. Sequences highly related to this alternative $\mathrm{N}$-terminus are also found in a number of other species. 
noted: an R295-Stop mutation in the coding region, and a Copia element inserted $3^{\prime}$ to the transcript. TUNEL assays and/or anti-active caspase immunostaining conducted using $e c^{\Delta 9}, e c^{E P \Delta 4}, e c^{\text {PlacZ }}$, and $e c^{56}$, confirmed a reduction in apoptosis in mid-pupal retinas (see Additional File 1).

Together, the above results strongly suggest that CG2904 is echinus. To test this definitively we asked if expression of CG2904 could rescue the echinus phenotype. We identified several cDNAs for CG2904 from a larval-pupal library (see below). One of these, designated ec-SF1, was introduced into flies under the control of the GMR promoter (GMR-ec-SF1 flies). These flies have wildtypeappearing eyes and IOC number (Fig. 2E,K), but when introduced into the echinus ecEP $\Delta 4$ background, GMR-ecSF1 restored adult ommatidial patterning and normal IOC cell death (Figure 2F,L). RH68894 represents a group of $3 \mathrm{~kb}$ cDNA species that overlap echinus on the antisense DNA strand. RH68894 is predicted to be a non-coding RNA, based on the lack of any reading frame of significant size. To test whether RH68894 has any role in mitigating the echinus phenotype, GMR-RH68894 was introduced into the fly (GMR-RH68894 flies). Adult eyes of GMR-RH68894 flies appear wildtype. In addition, when introduced into ecEP $\Delta 4$, GMR-RH68894 failed to rescue or alter the echinus rough eye phenotype. Together these observations demonstrate that CG2904 (hereafter simply referred to as echinus), and not RH68894, encodes echinus.

Pupal retinas from animals homozygous for new alleles of echinus such as $e c^{E P \Delta 4}, e c^{\Delta 9}$ and $e c^{56}$, as well as those from wildtype flies expressing GMR-CG2904-RNAi, showed a striking difference from retinas mutant for $e c^{1}$ (as obtained from the Bloomington Stock Center). $e c^{1}$ pupal eyes showed a significant increase in IOC number subsequent to the time when death normally occurs, and this increase was associated with only a modest level of side-

Table I: Average Number of IOCs

\begin{tabular}{|c|c|}
\hline Genotype & Average no. $10 \mathrm{C}$ \\
\hline$w-$ & 9.0 \\
\hline ec ${ }^{\prime}$ & $10.6 \pm 1.5$ \\
\hline GMR-ec $c^{R N A i}$ & $14.1 \pm 2.1$ \\
\hline ec EP $\Delta 4$ & $14.1 \pm 1.7$ \\
\hline GMR-ec (SFI) & $9.1 \pm 0.3$ \\
\hline ecEPA4; GMR-ec (SFI) & $9.6 \pm 1.0$ \\
\hline $\mathrm{ec}^{\prime}(\mathrm{A} 2 \mathrm{Cl})$ & $14.8 \pm 1.8$ \\
\hline $\mathrm{ec}^{\prime}(\mathrm{B} \mid \mathrm{BI})$ & $14.4 \pm 2.2$ \\
\hline $\mathrm{ec}^{3 \mathrm{c} 3}$ & $10.6 \pm 1.0$ \\
\hline
\end{tabular}

Table I shows the average number of IOCs for the genotypes indicated. Examples of adult and pupal eyes from these genotypes are shown in Fig. 2.
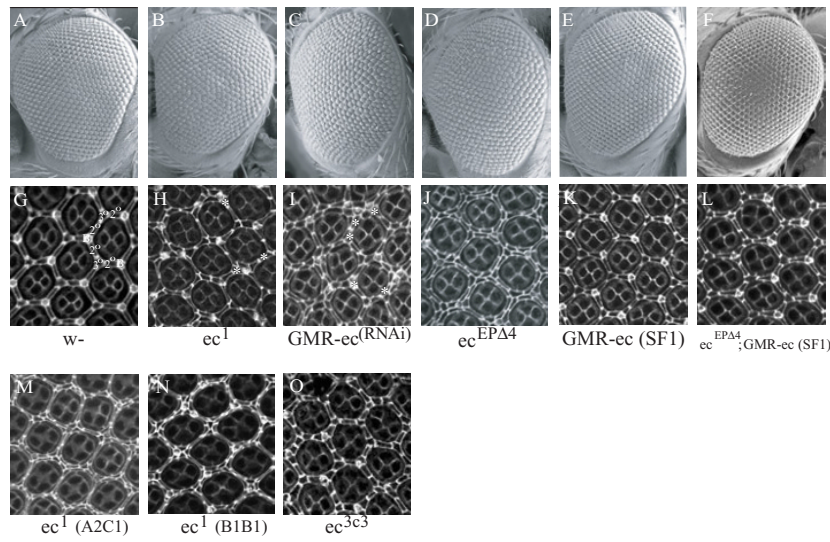

Figure 2

Flies with mutations in CG2904 have rough eyes, defects in IOC sorting, an increase in IOC number (A-F) SEM views of adult fly eyes of various genotypes. (G-O) Pupal retinas of various genotypes stained with anti-DIg. $(A, G)$ Wildtype flies have regularly spaced ommatidia and an invariant number of IOCs. Cell types indicated are bristle (B), $2^{\circ}, 3^{\circ}$, and asterisk represent extra IOCs. $(B, H)$ ec' flies obtained from the Bloomington Stock center have rough eyes and a modest number of extra $2^{\circ}$ and $3^{\circ}$ pigment cells. (C,I) GMR-driven RNAi of CG2904 results in flies with rough eyes and a large increase in IOCs, with many stacked side-by-side in parallel rows. (D,J) Flies homozygous for a deletion in CG2904, $e^{E P \Delta 4}$, have rough eyes, a large increase in IOCs, with many cells stacked side-by-side in parallel rows. (E,K) GMRdependent expression of ec-SFI has no effect on the adult eye and does not cause any excess death of IOCs. $(F, L)$ Expression of GMR-ec-SFI restores normal levels of IOC death to ec ${ }^{E P \Delta 4}$ flies. (M,N) Pupal eyes from two independent stocks of ecl outcrossed for 5 generations. There are increased numbers of IOCs as compared with the original ecl stock, and many extra cells are aligned side-by-side in parallel rows. (O) Pupal eyes from ec $\mathrm{ec}^{3 \mathrm{c}}$ flies have a modest increase in IOC number and few defects in cell sorting.

by-side alignment of IOCs (Fig. 2; Table 1) [2]. One of the new EMS alleles isolated, $e c^{3 c 3}$, which contains a stop codon near the C-terminus of the echinus coding region, showed a similar phenotype (Fig. 2O). $\mathrm{ec}^{3 \mathrm{c} 3}$ is likely to be a partial loss-of-function allele of echinus since $e c^{3 c 3} /$ Df(1)HC244 results in a stronger adult rough eye phenotype than that observed in homozygous $e^{3 c 3}$ flies (see Additional File 2). In contrast, pupal eyes from $e c^{E P \Delta 4}$ and GMR-CG2904-RNAi (Fig. 2), $e c^{\Delta 9}$ and $e c^{56}$ (data not shown) showed a greatly increased number of IOCs (Table 1) and many of these extra cells were aligned sideby-side. The Bloomington $e c^{1}$ stock was outcrossed to wildtype flies for 5 generations in two independent experiments. Interestingly, pupal eyes from both of these outcrossed lines showed an increase in the number of extra IOCs (Table 1) and IOC cell stacking (Fig. 2M,N). Importantly, both sorting and death phenotypes were rescued 
by GMR-dependent expression of ec-SF1 for multiple echinus alleles (Fig. 2). Together, these observations suggest that the original $e c^{1}$ line has picked up one or more suppressor mutations and that the true echinus null phenotype in the pupal eye results in extra IOCs, with many of these cells being stacked side-by-side. In addition, there seems to be a direct correlation between the severity of defects in sorting and those in cell death. Thus, pupal retinas from the original $e c^{1}$ allele and $e c^{3 c 3}$ displayed mild defects in sorting and IOC death, while retinas from the deletion allele e $c^{E P \Delta 4}$, outcrossed $e c^{1}$, GMR-CG2904-RNAi, and $e c^{E P \Delta 4} / \mathrm{Df}(1) \mathrm{HC} 244$ (a deficiency which covers the echinus locus) (data not shown), displayed much more severe defects in sorting and IOC death. These observations raise a question as to whether the echinus decreasein-IOC death phenotype is a result of loss of echinus function as a death activator, or a secondary consequence of a failure in sorting, which precludes death signaling (discussed further below).

cDNAs for echinus have been isolated from early embryos [54], as well as pupal eyes (this work), and genetic interactions between echinus and genes that result in phenotypes in tissues other than the eye have been described $[48,52]$. Thus, it is likely that echinus is expressed in, and plays roles in tissues other than the eye, though our focus in this work is the pupal eye. To determine the echinus expression pattern in this tissue we carried out tissue in situ hybridizations on pupal retinas with an antisense echinus cDNA probe. echinus transcripts could be detected at low levels in cone cells, primary pigment cells and IOCs prior to, and during the period of IOC death (Fig. 3A,B). The $e c^{\text {PlacZ }}$ allele carries a version of lacZ that functions as an enhancer trap. Therefore, as a second, and perhaps more sensitive method of visualizing echinus expression, we examined $\beta$-gal expression in pupal retinas from this line. Consistent with the results from echinus tissue in situ hybridizations, $\beta$-gal was expressed uniformly in cone cells, primary pigment cells and IOCs in wildtype (heterozygous $e c^{\text {PlacZ }}$ ) pupal retinas (Fig. 3C-E). These observations do not exclude the possibility that Echinus protein is differentially translated in specific cells, but they do suggest that transcription of echinus in specific populations of IOCs is not a critical point of cell sorting or cell death regulation.

To explore the question of where echinus expression was required during pupal eye development we took advantage of a GAL4-driver, LL54-GAL4, that is expressed predominantly, if not exclusively in primary, secondary and tertiary pigment cells, but not cone cells or bristles (Fig. 3F-G) [55]. Expression of LL54-GAL4 in a wildtype background, in conjunction with a UAS-driven miRNA (UASCG2904-RNAi) targeting all echinus splice forms, phenocopied the echinus phenotype (Fig. 3H). We cannot
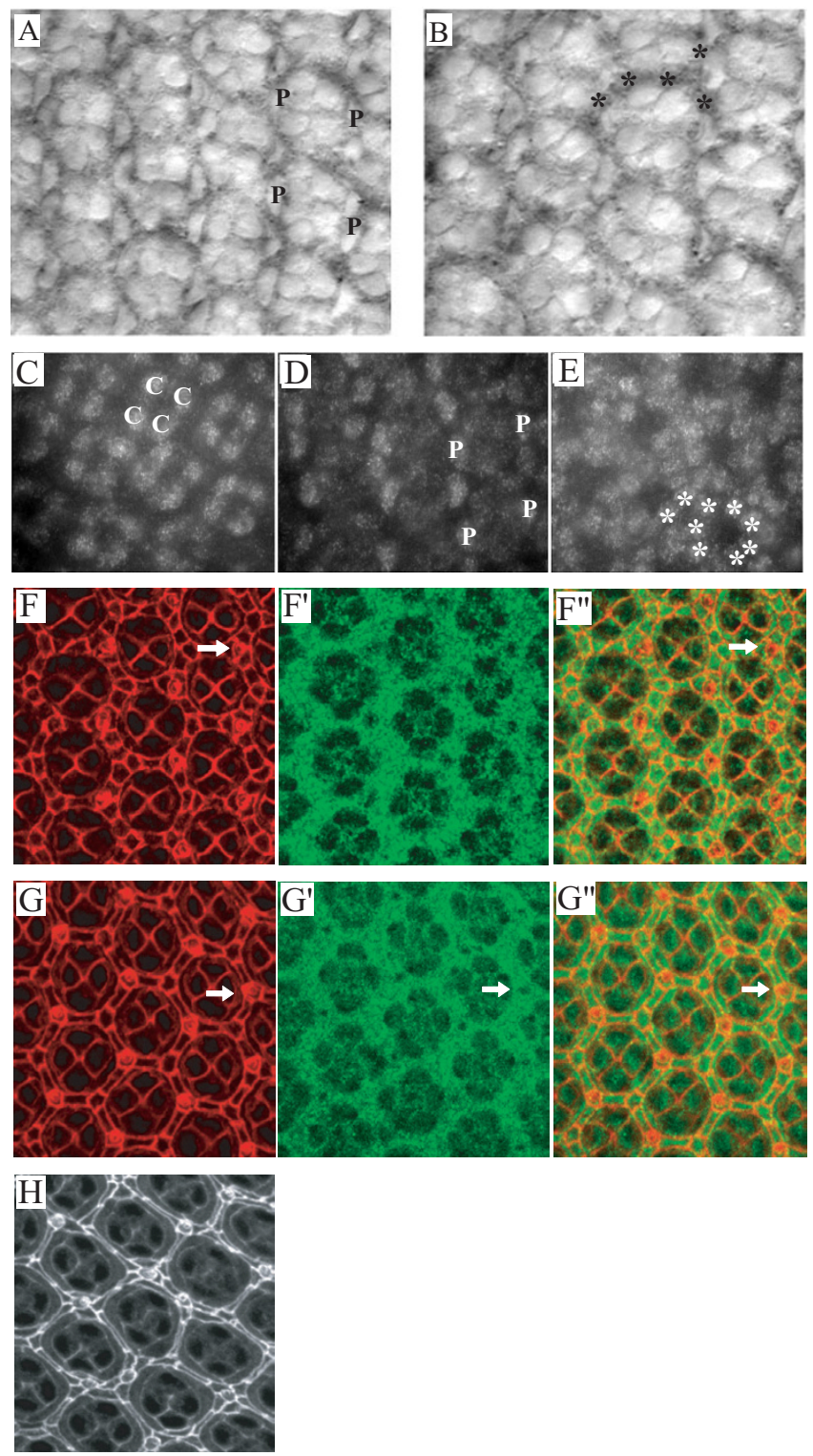

Figure 3

The echinus transcript is expressed at low, uniform levels in the pupal eye, and GAL4-driver-dependent expression of ec-SFI or an ec-silencing microRNA suggests that pigment cells are an important site of ec action. (A,B) Tissue in situ hybridization of an echinus antisense probe complementary to all splice forms in $28 \mathrm{hr}$ APF pupal retinas. (A) Focal plane showing primary pigment cells. (B) Focal plane showing IOCs. (C-E) Pupal retinas from heterozygous ec PlacZ/+ flies showing anti- $\beta$ galactosidase staining in cone cells $(C)$, primary pigment cells (D), and IOCs (E). Cell types indicated are cones (C), primaries (P), and IOCs (*). (F-F") $24 \mathrm{hr}$ pupal retina from LL54-GAL4; UAS-GFP flies stained with anti-Dlg to outline cell boundaries (F, F") and GFP ( $\left.F^{\prime}, F^{\prime \prime}\right)$ to visualize the LL54-GAL4 expression pattern. (G-G") $29 \mathrm{hr}$ pupal retinas from LL54-GAL4; UAS-GFP flies stained as above. LL54-GAL4 is expressed primarily, if not exclusively in pigment cells, but not bristles or cone cells. $(H) 36$ hr pupal eye from LL54-GAL4; UASCG2904-RNAi stained with anti-Dlg. Extra IOCs and sorting defects are apparent. Arrows indicate bristles. 
exclude the possibility that low-level expression of the echinus-targeting miRNA in other cell types in the eye is sufficient to generate this phenotype. This possibility notwithstanding, our observations suggest that echinus normally functions, at least in part, within the pigment cells to regulate IOC fate. Interestingly, however, expression of either of two different splice forms of echinus (ec-SF1 and ec-SF2; see below), in an $e c^{E P \Delta 4}$ background, under this same promoter, failed to rescue the e $c^{E P \Delta 4}$ phenotype (data not shown). This observation suggests, but does not prove, that echinus expression is also required in other cell types to bring about proper IOC sorting and death. Analysis of echinus clones will be required to determine definitively the cell types in which echinus expression is required. Finally, GMR-driven transgenes are expressed in all cell types in the developing eye [50,56,57]. As noted above (Fig. 2E,K), forced expression of echinus in all retinal cells did not by itself induce defects in sorting or ectopic retinal (IOC) cell death. This, in conjunction with the observation that endogenous echinus is expressed uniformly in the pupal retina, is consistent with a model in which echinus expression is not sufficient to induce the death of IOCs, though it is necessary.

\section{Echinus gives rise to multiple splice forms that encode proteins with homology to ubiquitin-specific proteases}

We sequenced multiple echinus cDNAs and identified three splice variants (designated ec-SF1, ec-SF2, and ecSF3) (Fig. 1A-C). In each of these, a common 3' coding and UTR sequence is spliced to distinct $5^{\prime}$ UTR and coding sequences. To determine which splice forms are expressed during pupal retinal cell death, we conducted RT-PCR using exon-specific primers. We found that all isoforms were expressed in the pupal retina during the stages when IOC death occurred (see Additional File 3). No differences were seen when expression of different splice forms was monitored using tissue in situ hybridizations (data not shown).

Each splice form encodes a large protein of roughly 1700 aa. Blast searches identified only one region of homology with other proteins, an N-terminal USP domain, a domain found in one of the seven families of deubiquitinating enzymes (DUBs). USP-containing DUBs are cysteine proteases that are capable of removing ubiquitin or ubiquitin-like proteins from substrates $[58,59]$. The USP domain features two short, well-conserved motifs the Cys box, which contains the essential catalytic cysteine, and a His box, which contains conserved His and Asp residues that are thought to be essential for catalysis. Structural studies on the USPs HAUSP and Ubp14 have revealed that the catalytic histidine and aspartic acid deprotonate the catalytic cysteine allowing for nucleophilic attack $[60,61]$. Ec-SF1 encodes an USP domain with all the known essential catalytic residues (Figure 1A).
Database searches identified genomic sequences that if spliced, would generate similar forms of echinus in multiple Drosophila species as well as several other insects. Outside of the arthropods Echinus shows most homology with the mammalian DUBs USP53 and USP54, with essentially all of this homology occuring within the USP domain. Most importantly for the purposes of this report, Ec-SF2 and Ec-SF3 encode proteins with truncated USP domains and lack residues important for catalysis. Specifically, Ec-SF2 and Ec-SF3 contain the catalytic histidine and aspartic acid residues found in the USP His box, but they lack the Cys box and its catalytic cysteine. Ec-SF2 and Ec-SF3 instead have alternative N-termini that are conserved in Drosophilia and show no sequence similarity to the Cys box motif (Figure 1B, C). The homologous mammalian DUB USP54 also encodes 4 alternative splice forms, two of which do not contain complete USP domains [59].

\section{Echinus lacks USP activity on a model substrate, and USP activity is not required for echinus-dependent death of IOCs}

We generated flies expressing a microRNA that targets EcSF1 specifically, under the control of the GMR promoter. These flies showed an echinus-like adult rough eye phenotype, and pupal eyes contained extra IOCs (Fig. 4A,E). In contrast, GMR-driven expression of microRNAs designed to target Ec-SF2 or Ec-SF3 resulted in flies that appeared wildtype, and no extra IOCs were observed (data not shown). We cannot exclude the possibility that the microRNAs targeting Ec-SF2 and Ec-SF3 failed to phenocopy echinus because they failed to induce a large enough decrease in splice form expression (though in other experiments expression of these miRNAs was sufficient to suppress rescue of echinus by GMR-dependent expression of ec-SF2 or ec-SF3; data not shown). Nonetheless, our results from targeting Ec-SF1 demonstrate that this splice form, at least, is important for bringing about IOC death, and suggest that it may be the most important for regulating IOC survival. Also consistent with this hypothesis is our observation that GMR-driven RNAi that targets all splice forms (GMR-CG2904-RNAi) results in a phenotype similar to that observed in flies in which only ec-SF1 was targeted (Fig 2C,I). USP activity per se is unlikely to be sufficient for rescue, since the echinus sorting and cell death phenotypes could not be rescued by GMR-dependent expression of yeast UBP2, a ubiquitin-specific protease known to be active on multiple substrates $[62,63]$ (data not shown).

To test the hypothesis that Ec-SF1 has deubiquitinating activity, we measured its ability to cleave a model ubiquitin-linked substrate, Ub-Arg-B-Gal, a fusion protein of Ubiquitin (Ub) and Eschericia coli $\beta$-galactosidase ( $\beta$-Gal), separated by an arginine (Arg) residue $[62,63]$. Ub-Arg- $\beta$ - 


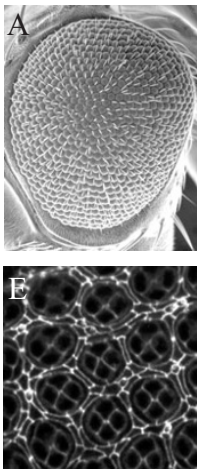

GMR-ec-SF1-RNAi
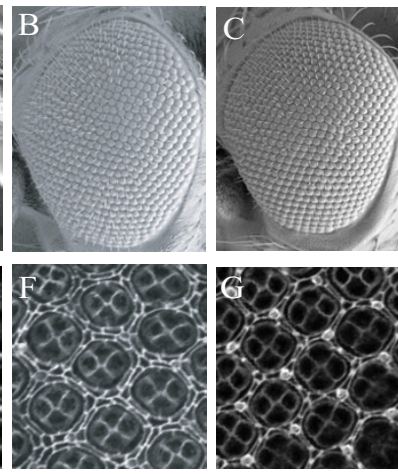

ec $\mathrm{EP} \Delta 4$
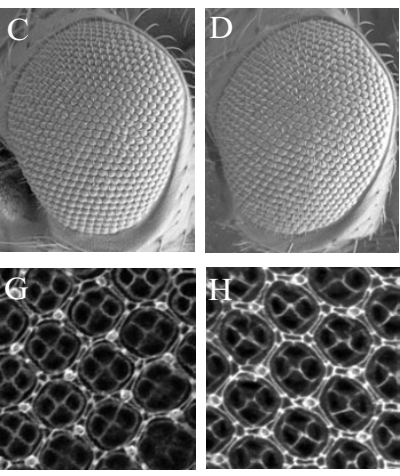

${ }^{\mathrm{EP} \Delta 4} ; \mathrm{GMR}-\mathrm{ec}-\mathrm{SF} 2$ ec ${ }^{\mathrm{EP} \Delta 4} ; \mathrm{GMR}-\mathrm{ec}-\mathrm{SF} 3$

\section{Figure 4}

Echinus does not require deubiquitinating activity to promote normal IOC death. (A-D) SEMs of adult eyes of various genotypes. $(E-H)$ Pupal retinas of various genotypes stained with anti-DIg. $(A, E)$ GMR-driven expression of a microRNA targeting ec-SFI results in an echinus phenotype. $(B, F)$ ec $E P \triangle 4$ eyes. $(C, G)$ Eyes of genotype ecEPA4; GMR-ec-SF2/+. (D,H) Eyes of genotype ecEPA4; GMR-ec-SF3/+. Expression of versions of Echinus that lack essential USP catalytic residues rescues the ec ${ }^{E P \Delta 4}$ phenotype.

gal is a stable protein, and thus bacterial cells expressing it form blue colonies in the presence of the substrate X-Gal (5-bromo-4-chloro-3-indolyl- $\beta$-D-galactopyranoside) (Table 2). Deubiquitinating enzymes that remove Ub create Arg- $\beta$-gal, an unstable protein. Thus, cells expressing Ub-Arg- $\beta$-gal, as well as an active deubiquitinating enzyme such as yeast Ubp2, give rise to white colonies in the presence of X-Gal (Table 2) [62]. Many deubiquitinat-

Table 2: Bacterial Assay for the Deubiquitinating Activity of Echinus

\begin{tabular}{|c|c|}
\hline Plasmid & Colony Color \\
\hline pUb-Arg- $\beta$-Gal & Blue \\
\hline pRBI05 (yUbp2) & White \\
\hline pRB-ec (SFI) & White \\
\hline pRB-ec (SF2) & White \\
\hline $\begin{array}{l}\text { pUb-Arg- } \beta \text {-Gal } \\
\text { pRBI } 05 \text { (yUbp2) }\end{array}$ & White \\
\hline $\begin{array}{l}\text { pUb-Arg- } \beta-\text { Gal } \\
\text { pRB-ec (SFI) }\end{array}$ & Blue \\
\hline $\begin{array}{l}\text { pUb-Arg- } \beta-\text { Gal } \\
\text { pRB-ec (SF2) }\end{array}$ & Blue \\
\hline
\end{tabular}

Table 2. Echinus ec-SFI lacks deubiquitinating activity on a model substrate in bacteria. Ubiquitin-Arg- $\beta$-Gal has a long half-life, and colonies expressing this protein alone are therefore blue in the presence of X-gal substrate. Expression of S. cerevisiae Ubp2 with Arg$\beta$-Gal results in cleavage of ubiquitin, exposing the $\mathrm{N}$-terminus of Arg$\beta$-Gal, which has a short half-life (white colonies). In contrast, expression of ec-SFI or ec-SF2 does not result in significant cleave Ub-Arg- $\beta$-Gal (blue colony color). ing enzymes are active in this assay. However, in contrast to yeast Ubp2, expression of ec-SF1 or ec-SF2 in Ub-Arg- $\beta$ gal cells, in the presence of X-Gal, resulted in the formation of blue colonies (Table 2). The human proteins most homologous to Ec-SF1, USP53 and USP54, also lack activity in this assay [64]. These results are consistent with models in which Echinus, in particular Ec-SF1, lacks deubiquitinating activity. However, as discussed below (the Discussion), the failure to detect cleavage of a model substrate does not rule out the possibility that Ec-SF1 has activity on other (unknown) substrates.

To test the hypothesis that Echinus USP activity is required for its ability to bring about the death of excess IOCs, we asked if expression of Echinus splice forms that lack critical USP catalytic residues, Ec-SF2 and Ec-SF3, could rescue the echinus phenotype. Somewhat to our surprise, when introduced into the ec ${ }^{E P \Delta 4}$ background (Fig. $4 \mathrm{~B}, \mathrm{~F}$ ), expression of Ec-SF2 (Fig. 4C,G) or Ec-SF3 (Fig. $4 \mathrm{D}, \mathrm{H})$, resulted in complete restoration of normal IOC death. These experiments involve gene overexpression and do not address the question of whether Ec-SF2 or EcSF3 is normally required for IOC death. However, they do demonstrate that Echinus USP activity is unlikely to be absolutely required for IOC death, since these splice forms lack residues necessary for this activity.

\section{Echinus does not show significant genetic interactions with components of the core apoptosis machinery, or other pathways implicated in regulation of retinal cell death}

Our observations presented in Fig. 2 indicate that null alleles of echinus have a previously unappreciated defect in cell sorting as well as cell death. The cell death defects observed in echinus mutants could simply be the indirect result of a failure in cell sorting. Alternatively, echinus may play roles in both sorting and cell death. We have chosen to explore how echinus could be promoting the death of specific IOCs. We searched for genetic interactions between echinus loss- and gain-of-function (overexpression) and signaling pathways known to regulate IOC death. GMR-ec phenotypes in Fig. 5 refer to ec-SF1. Similar phenotypes were observed with GMR-ec-SF2 (not shown). The RHG family protein Hid is required for normal IOC death, as are the caspases Dronc and Drice. Loss or overexpression of echinus had no significant effect on dominant eye phenotypes associated with GMR-driven overexpression of any of these molecules, or several other cell death activators including Rpr, Grim, Debcl (Fig. 5) or the caspases Dcp-1 and Strica (see Additional File 4). echinus loss- and gain-of-function also had no effect on a small eye phenotype associated with a partial loss-of-function in DIAP1 resulting from GMR-dependent expression of dsRNA corresponding to sequences within the diap1 coding region (GMR-diap1-RNAi flies) [12] (Fig. 5). 


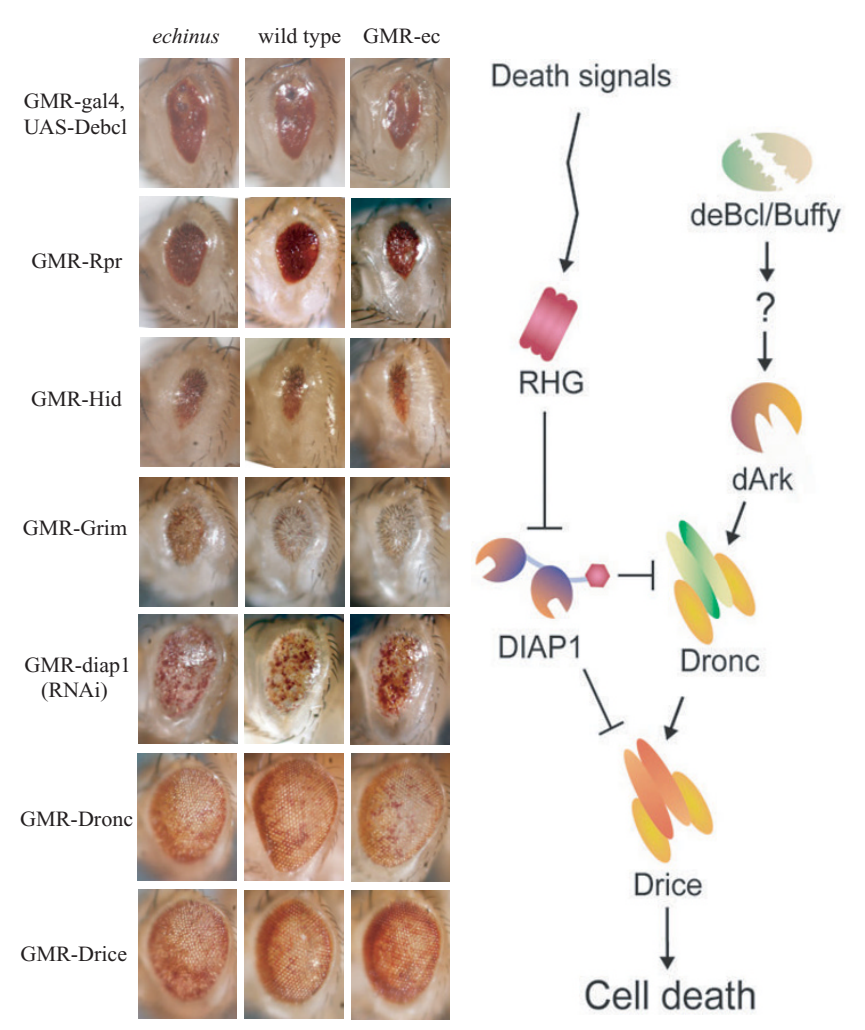

Figure 5

Genetic interactions between echinus and known or potential regulators of cell death in the eye. To the right is a schematic depicting known or suggested interactions between death regulators in the fly. The question mark separating Debcl/ Buffy from Ark indicates the uncertainy as to the roles these proteins play in regulating Ark activation or activity. GMRdriven transgenes of the indicated genotype were introduced into the ec ${ }^{E P \Delta} 4$ background, or into a wildtype background in the presence of GMR-ec-SFI. For each death regulator tested, similar phenotypes were observed in the presence of GMR-ec-SF2 (data not shown).

We also failed to see interactions between loss and gainof-function of echinus and mutations in several other pathways implicated in IOC death. These include the following: the EGF pathway (the dominant EGFR allele EGFREllipse, and GMR-driven versions of Ras, Sina and Yan); the Runx transcription factor lozenge $\left(l z^{50 e}\right.$ and GMR-lozenge); Notch (GMR-GAL4-UAS-Delta, Nfa-g); and JNK (GMR-GAL4, UAS-dTAK) (see Additional File 4). GMR-GAL4-UAS-klumpfuss was lethal in combination with GMR-ec. However, the significance of this interaction is unclear since expression of GMR-GAL4-UAS-klumpfuss alone resulted in only rare adults (see Additional File 4). Finally, no interactions were observed between loss- or gain-of-function mutations in echinus and rst (see Additional File 5).

\section{Discussion}

We showed that echinus, a gene required for normal IOC death, corresponds to CG2904. CG2904 generates multiple transcripts, each of which encodes a protein with homology to the USP family of ubiquitin-specific proteases. Echinus is necessary but not sufficient to cause IOC death when overexpressed. These results are consistent with models where echinus provides an activity that can modulate other signals that drive the death of specific cells (or prevent their survival), rather than providing death signals to specific cells. Previous analyses of the $e c^{1}$ allele led to a model wherein echinus functions subsequent to IOC sorting $[49,50]$. However, our analysis of multiple newly generated echinus alleles, including multiple likely null alleles, showed the presence of many excess IOC cells arranged in a side-by-side configuration with respect to primary pigment cells, in addition to excess cells arranged end-to-end. These observations are consistent with a model in which echinus functions to promote proper IOC sorting, with the failure in IOC death being a result of incorrectly positioned IOCs being unable to send or receive cell death signals. An alternative possibility that we explored is that echinus plays roles in cell death signaling as well as cell sorting. We failed to observe significant genetic interactions between echinus and known or suspected death regulators. While these observations do not rule out the possibility that echinus acts to regulate death at a novel point, they tend to support models in which echinus functions primarily to regulate cell sorting.

Homology searches of genome sequence in other insects suggest that echinus is conserved. But nothing is known about the functions of any of these genes. One splice form of echinus, ec-SF1, encodes a protein that contains catalytic residues essential for USP activity. ec-SF1 is expressed in the pupal eye and splice form-specific RNAi of this transcript phenocopied echinus. However, Ec-SF1 was inactive in a deubiquitination assay utilizing a model ubiquitin- $\beta$ gal fusion protein substrate. This may reflect the fact that ec-SF1 is inactive as a USP. Alternatively, Echinus may only be active on specific substrates, a phenomenon observed with a number of USPs $[58,59,65]$. It is also possible that Ec-SF1, as with several other USP family members, cleaves proteins modified with other ubiquitinrelated proteins such as ISG15 [66] or Nedd8 [67]. Regardless of the answer to this question, our observation that splice forms of Echinus that lack residues essential for USP activity rescue the echinus phenotype strongly suggests that ubiquitin or ubiquitin-like protease activity is not essential for Echinus function, at least for bringing about the sorting and death of excess IOCs. This does not mean that Echinus functions are necessarily unrelated to regulation of deubiquitination. Interestingly, like echinus, the genes USP53 and USP54 in human and mouse have a shorter splice form missing key catalytic residues and a 
longer form that includes these residues (the longer forms are shown in Fig. 1). Also like Echinus, the USP53 and USP54 long forms are inactive in a deubiquitination assay using a model ubiquitin- $\beta$-gal fusion protein substrate [63]. This conserved similarity in gene structure suggests a functional requirement for the multiple splice forms of echinus, despite the observed absence of protease activity. Inactive versions of known USPs can, in some cases, still bind ubiquitinated substrates, functioning as dominant negatives that block deubiquitination, thereby facilitating degradation or other events dependent on ubiquitin conjugation [68-71]. A number of components of the ubiquitin pathway regulate cell death in the fly eye, including the ubiquitin activating enzyme uba1, the $\mathrm{E} 3$ ubiquitin ligase DIAP1, two components of an SCF-type E3 ubiquitin ligase ( $s k p A$ and a novel F-box gene, morgue), and the deubiquitinating enzyme fat facets [29,31,32]. Perhaps Echinus promotes cell sorting and death by binding substrates modified with ubiquitin or ubiquitin-like proteins, thereby blocking the removal of these modifications. Alternatively, Echinus could titrate cellular inhibitors of ubiquitin or ubiquitin-like proteases. Finally, it is important to emphasize that Echinus's functions in sorting and cell death may be unrelated to ubiquitin or ubiquitin-like proteins. Some USPs affect signaling through pathways which are independent of their ability to remove ubiquitin or ubiquitin-like proteins [72]. Central to addressing these questions is the identification of proteins bound by Echinus in the eye.

As a first step in this direction we searched for genetic interactions between echinus and mutations in genes known or suspected to regulate IOC survival. EGFR or Notch, important upstream regulators of IOC survival. Particularly in the case of the Echinus overexpression experiments, our observations suggest that echinus is not sufficient on its own to regulate signaling through these pathways. We also searched for interactions between echinus and a number of known or suspected cell death effectors. These included Rpr, Hid, Grim, and the caspases Dronc, Drice, and Dcp-1. For each of these genes, and for the eye-specific partial loss-of-function of DIAP1 induced using GMR-diap1-RNAi, expression in the eye results in ectopic cell death. However, none of these phenotypes were significantly suppressed in the echinus loss-of-function background, or enhanced by echinus coexpression. Perhaps echinus does not regulate these components, or components in the same pathways, at downstream points. However, there are several caveats to this conclusion. First, echinus is rare at the level of mRNA, and thus presumably at the level of protein as well. Therefore, it may simply be that loss of echinus, which normally regulates its target(s) in the context of their much lower endogenous expression level, has little effect on phenotypes due to high-level target expression. Second, in the cases where Echinus was overexpressed, it may be that echinus requires cofactors in order to act on its targets, and these also may be relatively rare and rate-limiting. If true, overexpression of echinus might again be expected to have little effect on phenotypes associated with high-level expression of its target proteins. Therefore, our observations allow us to conclude at most that echinus is probably not a rate-limiting, or dose-dependent regulator of the above death activators.

\section{Conclusion}

The echinus locus encodes multiple splice versions of proteins with homology to USP family proteases. But there is no clear evidence that regulation of ubiquitination is relevant to echinus's role in promoting IOC sorting and death. Echinus did not show significant genetic interactions with a number of known death regulators, and expression of echinus was not sufficient on its own to induce ectopic death. Together these observations suggest several possibilities. The first is that echinus regulates - but only in very specific contexts/cell types - unknown (or untested) upstream regulators or effectors of the core cell death pathway. Alternatively, echinus may act as a necessary but not sufficient component in a parallel death signaling or effector pathway. Finally, echinus may function primarily to regulate cell sorting, with failure in this process leading to cell survival because IOCs are unable to effectively transmit or receive death signals. Drawing links between echinus and any of these pathways requires the identification of Echinus targets.

\section{Methods}

\section{Identification and sequencing of echinus alleles}

Adult males were exposed to EMS and mated with females carrying an attached-X chromosome (XX/Y). Progeny males that had rough eyes were crossed back to attached$\mathrm{X}$ females and stocks, designated $e c^{56}$ and $e c^{30}$, were established. Complementation tests were used to establish allelism of with echinus. Genomic DNA for each EMS allele was isolated from third instar larvae using standard DNA isolation protocols. The coding portions and flanking DNA of echinus were amplified by PCR using Platinum Taq polymerase (Invitrogen) and sequenced using the Big Dye terminator cycle sequencing kit (v3.1) on a 3730XL sequencer $(\mathrm{ABI})$. The obtained sequence was compared with the published Drosophila genomic sequence. Both strands were completely sequenced for each exon and any ambiguities or mutations were re-sequenced. The P element $e c^{P l a c Z}$ was isolated originally as a rough eye mutant mapping to the $\mathrm{X}$ chromosome that failed to complement $e c^{1}$ (BA Hay, unpublished). Excision alleles of this element, as well as those of a nearby element, EP(X)1343, were generated using standard techniques. Deletion alleles of echinus were generated through excision of $E P(X) 1343$. Approximately 300 independent excision lines were characterized using primers indicated in Figure 
1. Several deletions were identified that removed much of the echinus coding region. Two of these, $e c^{E P \Delta 4}$ and $e c^{\Delta 9}$, are indicated in Fig. 1A. The $e c^{3 c 3}$ allele was generated by EMS mutagenesis as previously described [53]. The $e c^{\Delta 9}$ deletion allele resulted from imprecise excision of a P element insertion within the adjacent gene roX1 (H. Kramer, unpublished).

\section{Isolation of echinus cDNAs}

echinus cDNAs were isolated from a larval-pupal cDNA library using a probe generated against the UCH domain of CG2904. Several clones were isolated and sequenced. These encoded two different splice forms of echinus, ecSF2 (Genbank AY576488) and ec-SF3 (Genbank DQ418878). To identify echinus 5' cDNA end sequences we carried out 5 ' RACE. Total RNA was isolated from $w^{1118}$ pupal eye discs using RNeasy Micro Kit (Qiagen). The 5' RACE System was used according to the manufacturer's protocol (Invitrogen). cDNA was made by reverse transcribing the pupal RNA using an echinus-specific primer (5'-GGTCTGCAGCTGCTGGAAAAGTTCC-3'). 5' echinus transcripts were isolated first by performing PCR using an anchor primer complementary to the dCTP cap of the cDNA and gene-specific primers (5'-AGATACAGTCTTGGCCACCGCATA-3', 5'-TTGTTGTTGGCGCTACTGCCATAGC-3', and 5'CCGCATTGATGCACCGATCCCTCTC-3'). Nested PCR followed using the internal echinus primers (5'-GAAACGATCGTCGAAAGGCGTCCAA-3', 5'-CCAGTGGGGCATGTGGCAGCGATGT-3', and 5'CCATTACGGCCAATTCCACGCTGCT-3') and another anchor primer. PCR amplicons were purified using QiaQuick Gel Extraction Kit (Qiagen) and sequenced. This work led to the identification of a third echinus splice form, ec-SF1 (Genbank DQ418877), which encodes distinct 5 ' noncoding and coding sequences.

\section{RNAi-mediated knockdown of echinus function}

We used two approaches to silence echinus expression. In the first approach a cDNA fragment was placed into the SympUAST vector, which carries UAS elements on opposite strands flanking the insert [73]. Transgenic flies carrying these constructs were recombined with GMR-Gal4 to generate GMR-Gal4-UAS-ec-RNAi flies. Several segments of the coding region were targeted with this strategy: residues 1237-1505, and 232-398, as defined with respect to the sequence of the ec-SF2 cDNA. We also generated flies expressing GMR-driven artificial microRNAs, using the mir-6.1 backbone, designed to target specific 21 bp sequences within echinus. In brief, 22 bp sequences complementary to echinus were substituted into the mir-6.1 precursor stem backbone at the position normally occupied by the mature mir- 6 miRNA (C.H. Chen and B.A. Hay, unpublished). The $22 \mathrm{nt}$ sequences targeted either the region surrounding the catalytic cysteine of ec-SF1(res- idues 562-583; cta agg gac tac tca atg gac c), ec-SF2 (residues 530-551; cta aga agt tct cga gca aaa c), or sequences common to all ec transcripts (SF1 residues 4252-4273; gca atg caa aaa tgg atg tag a). These constructs are known as GMR-ec-SF1-RNAi, GMR-SF2-RNAi, and GMRCG2904-RNAi, respectively.

\section{Echinus and RH68894 transgenes and expression}

The coding regions for two splice versions of echinus, ecSF2 and ec-SF3, that lack UCH domain catalytic residues, were introduced into GMR, generating GMR-ec-SF2 and GMR-ec-SF3, respectively. The coding region for ec-SF1, which contains all known UCH catalytic domain residues, was also introduced into GMR, generating GMR-ec-SF1.

\section{Drosophila lines and genetics}

Drosophila strains and crosses were performed at $25^{\circ} \mathrm{C}$. Pupal timing is expressed in hours, with the white prepupae stage defined as 0 hours after pupal formation (APF). Pupal dissections were performed at 36 hrs APF unless noted otherwise. The following strains were used: UASklumpfuss [74], Ellipse, $e c^{1}$, Notch ${ }^{\text {spl-1 }}$ (Bloomington Stock Center, Indiana University), GMR- $\Delta \mathrm{N}$ dcp-1 [75], GMRdrice [76], ec PlacZ (this work), GMR-hid, GMR-rpr [77], GMR-grim, GMR-dronc [6], GMR-strica and GMR-Gal4UAS-debcl [78] and GMR-Gal4-UAS-dTak1 [79]. The echinus deletion allele mutant $e c^{E P \Delta 4}$ was generated by imprecise excision of the P element insertion line EP(X)1343. LL54-GAL4-expressing flies were obtained from Craig Montell [55]. GMR-yUbp2 (GenBank M94916) was cloned into NotI-StuI of pGMR-1N. RH68894 (Research Genetics/Invitrogen) was cloned into the GMR vector.

\section{Echinus expression pattern}

A 1.2 kilobase region within echinus (residues 1,532 to 2,691 with respect to ec-SF2) was amplified by PCR using T3/T7-tailed primers. Digoxigenin-labeled RNA probes were prepared for both sense and antisense strands (Roche). In situ hybridization to OreR pupal retinas was performed essentially as described [80]. Pupal retinas from $e c^{\Delta 9}$ were used as a negative control for staining.

\section{RT-PCR analysis of echinus splice form expression}

Retinas from OreR pupae (26-27 hrs APF) were dissected into PBS and then transferred immediately to RNAlater (Ambion). Total RNA was extracted using Trizol (Invitrogen). RT-PCR Reactions were performed $(n=3)$ using the SYBR Green One-step RT-PCR reagent kit on an Applied Biosystems 7900 Sequence Detection System. Each $15 \mu \mathrm{l}$ reaction included 50ng of total RNA and $0.1 \mu \mathrm{M}$ of each primer. The echinus and rp49 primer pairs were designed using Primer Express Version 2.0 software (Applied Biosystems) and were constructed to span an intron. Gel electrophoresis melting curve analysis was performed for each run to ensure there was a single major product corre- 
sponding to the predicted size and melting temperature. Primer sequences: SF1 splice form: 5'-TGCTTTCTCAATTGTGCCGT-3', SF2 splice form: 5'-CAACATTGGCGCATTCTTTC-3', common $3^{\prime}$ primer for SF1 and SF2: 5'AAGATACAGTCTTGGCCACCG-3', SF3 splice form: 5'GCCTTGTGCCTGCAAAAGTT-3', 5'-TCAGAGTCACAACATGGCAGC-3', all echinus splice forms: 5'-CAGCTGCCCTTCACCCA-3', 5'-TATGTCGCCCATGTTGCC-3', rp49: 5'-AGTCGGATCGATATGCTAAGCT-3', 5'-AGATACTGTCCCTTGAAGCGG-3'.

\section{Microscopy, immunocytochemistry, and antibodies}

Scanning electron microscope images were produced on a Hitachi machine. Flies were dehydrated in an ethanol series, incubated in hexamethyldisilazane (Sigma) overnight, and dried prior to use. Pupal retinas were dissected in PBS and fixed for 30 minutes in 4\% paraformaldehyde. Immunostaining was carried out in PBT (PBS $+0.1 \%$ Triton-X100) containing $10 \%$ fetal calf serum. Antibodies were used at the following concentration: mouse anti-Dlg $(1: 150)$ and mouse anti- $\beta$ galactosidase (1:15) (Developmental Studies Hybridoma Bank, University of Iowa, Iowa City, IA), rabbit cleaved caspase-3 (1:100, Cell Signaling Technologies). Secondary antibodies included mouse Alexa Fluor 488 (Molecular Probes) and mouse or rabbit IgG conjugated to Cy3 (Jackson Labs). Pupal retinas were mounted in either VectaShield medium (Vector, Burlingome, CA) or Antifade (Molecular Probes).

\section{TUNEL staining}

Pupal retinas were dissected in PBS and fixed for $30 \mathrm{~min}$ utes in 3\% paraformaldehyde. The In situ Cell Death Detection Kit (Roche Applied Science) or Promega DeadEnd Fluorometric TUNEL system was used for TUNEL labeling with fluorescein-dUTP. Tissues were incubated at $37^{\circ} \mathrm{C}$ for 1 hour in the mixture of enzyme and label solution then rinsed in PBS. Labeled tissues were mounted in Antifade reagent (Molecular Probes), viewed with a Zeiss Axioplan 2 and images were captured using a Retiga 1350EX digital camera (Qimaging Corp.) and Northern Eclipse software (Empix Imagin, Inc.).

\section{Interommatidial cell counts}

Interommatidial cell counts were made by counting the IOCs, minus the bristles, that surround two primary pigment cells. Three separate areas were counted per pupal retina, and at least five pupal retinas were counted for each genotype.

\section{Deubiquitination assay}

Deubiquitination assays were carried out as described in [62].

\section{Authors' contributions}

JMC carried out most of the experiments in this study. IB and JDF isolated and sequenced echinus alleles, and carried out tissue-in-situ hybridization and RT-PCR analysis of echinus expression. MG participated in data analysis, experimental design and writing of manuscript. SG and $\mathrm{BAH}$ were responsible for overall experiment design, analysis of data and, in conjunction with JMC, writing of the manuscript. All authors read and approved the final manuscript.

\section{Additional material}

\section{Additional file 1}

echinus mutants have a decrease in IOC apoptosis. TUNEL staining of (A) OreR, (B) ec ${ }^{\text {PlacZ }}$ and (C) ec ${ }^{49}$ pupal retinas (29-30 hr APF). Antiactive caspase-3 immunostaining in (D) OreR and (E) ec ${ }^{56}$ and $(F)$ ec ${ }^{\mathrm{EP}} 44$ pupal retinas (30 hr APF). Apoptosis is reduced, though not completely absent, in ec mutant pupal retinas.

Click here for file

[http://www.biomedcentral.com/content/supplementary/1471213X-7-82-S1.pdf]

\section{Additional file 2}

Phenotype of the $\mathrm{ec}^{3 \mathrm{c} 3}$ allele in trans to a deficiency for the region containing echinus. Scanning electron micrographs and pupal retinas of several different genotypes are shown. $\mathrm{ec}^{3 \mathrm{c} 3}$ placed in trans to a deficiency that removes echinus, $\mathrm{Df}(1) \mathrm{HC} 244$, shows a more severe rough eye phenotype than homozygous $\mathrm{ec}^{3 \mathrm{c}}$ flies. Pupal retinas show a significant increase in the number and improper sorting of IOCs. This genetic observation suggests ec ${ }^{3 c 3}$ represents a partial loss-of-function allele. Click here for file

[http://www.biomedcentral.com/content/supplementary/1471213X-7-82-S2.pdf] 


\section{Additional file 3 \\ Three echinus splice forms, ec-SF1, ec-SF2 and ec-SF3, are expressed in the pupal retina during the stage of IOC death. Gel image shows the results of RT-PCR analysis using primers for a positive control (rp49), primers specific to each echinus splice form (SF1,SF2, SF3), and primers that recognize all three splice forms (all). $M=$ Size Marker; - and + des- ignate the absence or presence of pupal retinal RNA template; expected product sizes are as follows: $91 \mathrm{bp}$ (rp49), $104 \mathrm{bp}$ (SF1), $111 \mathrm{bp}$ (SF2), $147 \mathrm{bp}$ (SF3), $135 \mathrm{bp}$ (all). \\ Click here for file \\ [http://www.biomedcentral.com/content/supplementary/1471- 213X-7-82-S3.pdf]}

\section{Additional file 4}

Genetic interactions between echinus and components of several signaling pathways. The indicated genotypes were introduced into the ec ${ }^{\Delta 4} \mathrm{back}$ ground, or into a wildtype background in the presence of GMR-ec-SF1. For each genotype tested, similar phenotypes were observed in the presence of GMR-ec-SF2 (data not shown). Genotypes not discussed in the text are indicated below. The EGFR ${ }^{\mathrm{ELP}}$ mutation is a hypermorphic allele of the EGF receptor $[81,82]$. However, genetically it behaves as a partial loss-offunction allele in the eye because it induces the expression of high levels of the EGFR inhibitor Argos [83]. Downstream Ras pathway components tested include Ras ${ }^{\mathrm{N} 17}$ (a dominant negative version of Ras driven by the sevenless promoter) Sina and Yanact (a version of Yan that is not inhibited by MAPKinase phosphorylation). GMR-GAL4-UAS-Delta expresses the Notch ligand Delta in every cell behind the morphogenetic furrow [84]. $\mathrm{N}^{\mathrm{fa}-\mathrm{g}}$ removes Notch activity specifically in pigment cells [85], leading to a failure of $1^{\circ}$ pigment cells to differentiate, and a decrease in IOC death [86]. Notch and echinus are both on the X chromosome. To search for interactions between $\mathrm{NaA-g}$ and echinus loss-of-function mutations we took advantage of flies carrying an autosomal insertion of GMR-CG2904RNAi that targets transcript sequences common to all echinus splice forms, and that phenocopies echinus (Fig. 2C,I). GMR- $\triangle \mathrm{N}-\mathrm{DCP} 1$ expresses under GMR control a version of the caspse DCP-1 that lacks the $\mathrm{N}$-terminal prodomain. GMR-GAL4-UAS-dTAK flies express under GMR control the kinase TAK1, an activator of JNK signaling. GMR-Strica flies express the long prodomain caspase under GMR control.

Click here for file

[http://www.biomedcentral.com/content/supplementary/1471213X-7-82-S4.pdf]

\section{Additional file 5}

echinus does not interact with roughest. Eye-specific Gain-(GMRecSF1) and loss-of-function (GMR-ec-RNAi) of echinus mutants were introduced into gain (GMR-GAL4-UAS-rst) and loss-of-function (rst ${ }^{\mathrm{CT}}$ ) roughest mutant backgrounds. No significant interactions were observed between these genes.

Click here for file

[http://www.biomedcentral.com/content/supplementary/1471213X-7-82-S5.pdf]

\section{Acknowledgements}

We thank Burke Judd and Helmut Kramer for sharing unpublished information, Helmut Kramer and Ting Wu for echinus stocks, and Marco Marra for helpful discussions. SG is grateful to the BC Cancer Foundation and NSERC (Discovery Grant \#250I25 to M. Marra) for financial support. Financial support was provided by $\mathrm{NIH}$ grant GM057422 to B.A.H. and by NIH grants NS042580 and NS048396 to M.G.

\section{References}

I. Cagan RL, Ready DF: The emergence of order in the Drosophila pupal retina. Dev Biol 1989, I36(2):346-362.

2. Wolff $T$, Ready DF: Cell death in normal and rough eye mutants of Drosophila. Develop I 99 I, I I 3:825-839.

3. Hay BA, Guo M: Caspase-dependent cell death in Drosophila. Annu Rev Cell Dev Biol 2006, 22:623-650.

4. Shi Y: Caspase activation, inhibition, and reactivation. Protein Science 2004, I 3:1979-1987.

5. Meier P, Silke J, Leevers SJ, Evan GI: The Drosophila caspase DRONC is regulated by DIAPI. Embo J 2000, I 9(4):598-6II.

6. Hawkins CJ, Yoo SJ, Peterson EP, Wang SL, Vernooy SY, Hay BA: The Drosophila caspase DRONC cleaves following glutamate or aspartate and is regulated by DIAPI, HID, and GRIM. J Biol Chem 2000, 275(35):27084-27093.

7. Chew SK, Akdemir F, Chen P, Lu WJ, Mills K, Daish T, Kumar S, Rodriguez A, Abrams JM: The apical caspase dronc governs programmed and unprogrammed cell death in Drosophila. Developmental Cell 2004, 7:897-907.

8. Daish TJ, Mills K, Kumar S: Drosophila caspase Dronc is required for specific developmental cell death pathways and stressinduced apoptosis. Developmental Cell 2004, 7:909-915.

9. Xu D, Li Y, Arcaro M, Lackey M, Bergmann A: The CARD-carrying caspase Dronc is essential for most, but not all, developmental cell death in Drosophila. Development 2005, I 32:2 I 25-2I 34.

10. Yu SY, Yoo SJ, Yang L, Zapata C, Srinivasan A, Hay BA: A pathway of signals regulating effector and initiator caspases in the developing Drosophila eye. Development 2002, I 29:3269-3278.

II. Xu D, Wang Y, Willecke R, Chen Z, Ding T, Bergmann A: The effector caspases drICE and dcp-I have partially overlapping functions in the apoptotic pathway in Drosophila. Cell Death Differ 2006, 13:1697-1706.

I2. Muro I, Berry DL, Huh JR, Chen CH, Huang H, Seoul JH, Yoo SJ, Guo $\mathrm{M}$, Baehrecke EH, Hay BA: The Drosophila caspase Ice is important for many apoptotic cell deaths and for spermatid individualization, a nonapoptotic process. Development 2006, I33:3305-3315

13. Wang SL, Hawkins CJ, Yoo SJ, Muller HA, Hay BA: The Drosophila caspase inhibitor DIAPI is essential for cell survival and is negatively regulated by HID. Cell 1999, 98(4):453-463.

14. Hawkins C], Wang SL, Hay BA: A cloning method to identify caspases and their regulators in yeast: identification of Drosophila IAP I as an inhibitor of the Drosophila caspase DCP. I. Proc Natl Acad Sci U S A 1999, 96(6):2885-2890.

I5. Wilson R, Goyal L, Ditzel M, Zachariou A, Baker DA, Agapite J, Steller $H$, Meier P: The DIAPI RING finger mediates ubiquitination of Dronc and is indispensable for regulating apoptosis. Nature Cell Biology 2002, 4(6):445-450.

16. Chai J, Yan N, Huh JR, Wu JW, Li W, Hay BA, Shi Y: Molecular mechanisms of Reaper-Grim-Hid-mediated suppression of DIAPI-dependent Dronc ubiquitination. Nature Structural Biology 2003, I 0:892-898.

17. Zachariou A, Tenev T, Goyal L, Agapite J, Steller H, Meier P: IAPantagonists exhibit non-redundant modes of action through differential DIAPI binding. EMBO J 2003, 22:6642-6652.

18. Yan N, Wu JW, Chai J, Li W, Shi Y: Molecular mechanisms of Drice inhibition by DIAPI and removal of inhibition by Reaper, Hid and Grim. Nature Structural and Molecular Biology 2004, I I:420-428.

19. Tenev T, Zachariou A, Wilson R, Ditzel M, Meier P: IAPs are functionally non-equivalent and regulate effector caspases through distinct mechanisms. Nature Cell Biology 2005, 7:70-77.

20. White K, Grether ME, Abrams JM, Young L, Farrell K, Steller H: Genetic control of programmed cell death in Drosophila. Science 1994, 264(5 I 59):677-683.

21. Grether ME, Abrams JM, Agapite J, White K, Steller H: The head involution defective gene of Drosophila melanogaster functions in programmed cell death. Genes Dev 1995 9(14): 1694-1708.

22. Chen P, Nordstrom W, Gish B, Abrams JM: grim, a novel cell death gene in Drosophila. Genes Dev 1996, I0( I4): I773-I782.

23. Christich A, Kauppila S, Chen P, Sogame N, Ho SI, Abrams JM: The Damage-Responsive Drosophila Gene sickle Encodes a Novel IAP Binding Protein Similar to but Distinct from reaper, grim, and hid. Curr Biol 2002, I2(2): I37-I 40. 
24. Srinivasula SM, Datta $P$, Kobayashi M, Wu JW, Fujioka M, Hegde R, Zhang Z, Mukattash R, Fernandes-Alnemri T, Shi Y, Jaynes JB, Alnemri ES: sickle, a Novel Drosophila Death Gene in the reaper/hid/ grim Region, Encodes an IAP-Inhibitory Protein. Curr Bio 2002, I 2(2): 125-130.

25. Wing JP, Karres JS, Ogdahl JL, Zhou L, Schwartz LM, Nambu JR: Drosophila sickle Is a Novel grim-reaper Cell Death Activator. Curr Biol 2002, I 2(2): | 3 |- I 35 .

26. Tenev T, Zachariou A, Wilson R, Paul A, Meier P: Jafrac2 is an IAP antagonist that promotes cell death by liberating Dronc from DIAPI. Embo J 2002, 2 I (19):51 I8-5 I 29

27. Kurada $P$, White K: Ras promotes cell survival in Drosophila by downregulating hid expression. Cell 1998, 95(3):319-329.

28. Yoo SJ, Huh JR, Muro I, Yu H, Wang L, Wang SL, Feldman RMR, Clem RJ, Muller HAJ, Hay BA: Apoptosis inducers Hid, Rpr and Grim negatively regulate levels of the caspase inhibitor DIAPI by distinct mechanisms. Nature Cell Biol 2002, 4:4I 6-424.

29. Hays R, Wickline L, Cagan R: Morgue mediates apoptosis in the Drosophila melanogaster retina by promoting degradation of DIAP I. Nature Cell Biology 2002, 4(6):425-43I.

30. Holley CL, Olson MR, Colon-Ramos DA, Kornbluth S: Reaper eliminates IAP proteins through stimulated IAP degradation and generalized translational inhibition. Nature Cell Biol 2002 , 4:439-444.

31. Ryoo HD, Bergmann A, Gonen H, Ciechanover A, Steller H: Regulation of Drosophila IAPI degradation and apoptosis by reaper and ubcDI. [erratum appears in Nat Cell Biol 2002 Jul;4(7):546.]. Nature Cell Biology 2002, 4(6):432-438.

32. Wing JP, Schreader BA, Yokokura T, Wang Y, Andrews PS, Huseinovic N, Dong CK, Ogdahl JL, Schwartz LM, White K, Nambu JR: Drosophila Morgue is an $F$ box/ubiquitin conjugase domain protein important for grim-reaper mediated apoptosis. Nat Cell Biol 2002, 4(6):45I-456.

33. Olson MR, Holley CL, Yoo SJ, Huh JR, Hay BA, Kornbluth S: Reaper is regulated by IAP-mediated ubiquitination. J Biol Chem 2002 , 278:4028-4034.

34. Yoo SJ: Grim stimulates DIAP I poly-ubiquitination by binding to UbcDI. Mol Cells 2005, 20:446-45I.

35. Ramos RGP, Igloi GL, Lichte B, Baumann U, Maier D, Schneider T, Brandstatter JH, Frohlich A, Fischbach KF: The irregular chiasm C. roughest locus of Drosophila, which affects axonal projections and programmed cell death, encodes a novel immunoglobulin-like protein. Genes Dev 1993, 7:2533-2547.

36. Gorski SM, Baker Brachmann C, Tanenbaum SB, Cagan RL: Delta and Notch promote correct localization of IrreC-rst. Cell Death and Differentiation 2000, 7:1011-1013.

37. Bao S, Cagan R: Preferential adhesion mediated by Hibris and Roughest regulates morphogenesis and patterning in the Drosophila eye. Developmental Cell 2005, 8:925-935

38. Grzeschik NA, Knust E: IrreC/rst-mediated cell sorting during Drosophila pupal eye development depends on proper localization of DE-cadherin. Development 2005, I 32:2035-2045.

39. Freeman M: Reiterative use of the EGF receptor triggers differentiation of all cell types in the Drosophila eye. Cell 1996, 87:65I-660.

40. Miller DT, Cagan RL: Local induction of patterning and programmed cell death in the developing Drosophila retina. Development 1998, 1 25:2327-2335.

4I. Bergmann A, Agapite J, McCall K, Steller H: The Drosophila gene hid is a direct molecular target of Ras-dependent survival signaling. Cell I998, 95(3):33|-34I.

42. Dikic I: Distinct monoubiquitin signals in receptor endocytosis. Trends in Biochemical Sciences 2003, 28:598-604.

43. Le Borgne $R$, Bardin A, Schweisguth F: The roles of receptor and ligand endocytosis in regulating Notch signaling. Development 2005, I 32: $1751-1762$.

44. Wildonger J, Sosinsky A, Honig B, Mann RS: Lozenge directly activates argos and klumpfuss to regulate programmed cell death. Genes and Development 2005, I9:1034-1039.

45. Siddall NA, Behan KJ, Crew J R., Cheung TL, Fair JA, Batterham P, Pollock JA: Mutations in lozenge and D-Pax2 invoke ectopic patterned cell death in the developing Drosophila eye using distinct mechanisms. Dev Genes Evol 2003, 213:107-119.

46. Flores GV, Daga A, Kalhor HR, Banerjee U: Lozenge is expressed in pluripotent precursor cells and patterns multiple cel types in the Drosophila eye through the control of cell-specific transcription factors. Development 1998, I 25:368I-3687.

47. Rusconi JC, Fink JL, Cagan R: klumpfuss regulates cell death in the Drosophila retina. Mechanisms of Development 2004 | 2 |:537-546.

48. Lindsley DL, Zimm GG: The genome of Drosophila melanogaster. San Diego, Academic Press; 1992

49. Hay BA, Wolff T, Rubin GM: Expression of baculovirus P35 prevents cell death in Drosophila. Development 1994 I 20(8):2| 2|-2|29.

50. Reiter C, Schimansky T, Nie Z, Fischbach KF: Reorganization of membrane contacts prior to apoptosis in the Drosophila retina: the role of the IrreC-rst protein. Development 1996, I 22(6): 1931 - 1940

5I. Baker Brachmann C, Cagan RL: Patterning the fly eye: the role of apoptosis. Trends in Genetics 2003, 19:91-96.

52. Morris JR, Chen J, Filandrinos ST, Dunn RC, Fisk R, Geyer PK, Wu C: An analysis of transvection at the yellow locus of Drosophila melanogaster. Genetics 1999, 151:633-651.

53. Manak JR, Dike S, Sementchenko V, Kapranov P, Biemar F, Long J, Cheng J, Bell I, Ghosh S, Piccolboni A, Gingeras TR: Biological function of unannotated transcription during the early development of Drosophila melanogaster. Nature Genetics 2006 38: $|15|-|| 58$.

54. Wang T, Montell C: Rhodopsin formation in Drosophila is dependent on the PINTA retinoid-binding protein. I Neurosci 2005, 25:5187-5194

55. Moses K, Ellis MC, Rubin GM: The glass gene encodes a zinc-finger protein required by Drosophila photoreceptor cells. Nature 1989, 340:531-536.

56. Ellis MC, O'Neill EM, Rubin GM: Expression of Drosophila glass protein and evidence for negative regulation of its activity in non-neuronal cells by another DNA-binding protein. Development 1993, I | 9(3):855-865

57. Amerik AY, Hochstrasser M: Mechanism and function of deubiquitinating enzymes. Biochemica et Biophysica Acta 2004 I 695: | 89-207.

58. Nijman SMB, Luna-Vargas MPA, Velds A, Brummelkamp TR, Dirac AMG, Sixma TK, Bernards R: A genomic and functional inventory of deubiquitinating enzymes. Cell 2005, I 23:773-786.

59. Baker RT, Tobias JW, Varshavsky A: Ubiquitin-specific proteases of Saccharomyces cerevisiae: Cloning of UBP2 and UBP3, and functional analysis of the UBP family. J Biol Chem 1992 267:23364-23375.

60. Huang Y, Baker RT, Fischer-Vise JA: Control of cell fate by a deubiquitinating enzyme encoded by the fat facets gene. Science 1995, 270:|828-|83|.

61. Quesada V, Diaz-Perales A, Gutierrez-Fernandez A, Garabaya C, Ca $S$, Lopez-Otin C: Cloning and enzymatic analysis of $\mathbf{2 2}$ novel human ubiquitin-specific proteases. BBRC 2004, 3 I 4:54-62.

62. Amerik AY, Li S], Hochstrasser M: Analysis of the deubiquitinating enzymes of the yeast Saccharomyces cerevisiae. Biol Chem 2000, 381:981-992.

63. Malakhov MP, Malakhova OA, Kim KI, Ritchie KJ, Zhang DE: UBP43 (USPI8) specifically removes ISGI5 from conjugated proteins. I Biol Chem 2002, 277:9976-998I.

64. Gong L, Kamitani T, Millas S, Yeh ET: Identification of a novel isopeptidase with dual specificity for ubiquitin- and NEDD8conjugated proteins. I Biol Chem 2000, 275:|42|2-|42|6.

65. Naviglio S, Mattecucci C, Matoskova B, Nagase T, Nomura N, Di Fiore PP, Draetta GF: UBPY: A growth-regulated human ubiquitin isopeptidase. EMBO J 1998, I 7:324|-3250.

66. DeSalle LM, Pagano M: Regulation of the GI to S transition by the ubiquitin pathway. FEBS Lett 2001, 490:179-189.

67. Lopez-Otin C, Overall CM: Protease degradomics: a new challenge for proteomics. Nat Rev Mol Cell Biol 2002, 3:509-5I9.

68. Li M, Chen D, Shiloh A, Luo J, Nikolaev AY, Qin J, Gu W: Deubiquitination of p53 by HAUSP is an important pathway for p53 stabilization. Nature 2002, 4 I 6:648-653.

69. Malakhova OA, Kim KI, Luo JK, Zou W, Kumar KGS, Fuchs SY, Shua $K$, Zhang DE: UBP43 is a novel regulator of interferon signaling independent of its ISG I5 isopeptidase activity. EMBO 2006, 25:2358-2367.

70. Giordano E, Rendina R, Peluso I, Furia M: RNAi Triggered by Symmetrically Transcribed Transgenes in Drosophila melanogaster. Genetics 2002, I 60(2):637-648. 
7I. Klein T, Campos-Ortega JA: Klumpfuss, a Drosophila gene encoding a member of the EGR family of transcription factors, is involved in bristle and leg development. Development 1997, 124:3 I23-3134.

72. Xu P, Vernooy SY, Guo M, Hay BA: The Drosophila microRNA mir-14 suppresses cell death and is required for normal fat metabolism. Current Biology 2003, I 3:790-795.

73. Huh JR, Foe I, Chen CH, Guo M, Park JM, Hay BA: The Drosophila Inhibitor of Apoptosis DIAP2 is required for the innate immune response to Gram-negative bacterial infection, and can be negatively regulated by IAP-binding proteins of the Reaper/Hid/Grim family. J Biol Chem 2006, 282:2056-2068.

74. Hay BA, Wassarman DA, Rubin GM: Drosophila homologs of baculovirus inhibitor of apoptosis proteins function to block cell death. Cell I995, 83(7): I253-I262.

75. Vernooy SY, Chow V, Su J, Verbrugghe K, Yang J, Cole S, Olson MR, Hay BA: Drosophila Bruce can potently suppress Rpr- and Grim-dependent but not Hid-dependent cell death. Curr Biol 2002, I 2:1164-1168.

76. Takatsu $Y$, Nakamura M, Stapleton M, Danos MC, Matsumoto K, O'Connor MB, Shibuya H, Ueno N: TAKI participates in c-Jun Nterminal kinase signaling during Drosophila development. Mol Cell Biol 2000, 20:30I5-3026.

77. Tautz $D$, Pfeifle $C:$ A non-radioactive in situ hybridization method for the localization of specific RNAs in Drosophila embryos reveals translational control of the segmentation gene hunchback. Chromosoma 1989, 98(2):8I-85.

78. Baker NE, Rubin GM: Effect on eye development of dominant mutations in Drosophila homologue of the EGF receptor. Nature 1989, 340:150-153.

79. Lesokhin AM, Yu SY, Katz J, Baker NE: Several levels of EGF receptor signaling during photoreceptor specification in wild-type, Ellipse, and null mutant Drosophila. Dev Biol 1999, 205: 129-| 44.

80. Spencer SA, Powell PA, Miller DT, Cagan RL: Regulation of EGF receptor signaling establishes pattern across the developing Drosophila retina. Development 1998, I 25:4777-4790.

8I. Shellenbarger DL, Mohler JD: Temperature-sensitive mutations of the Notch locus in Drosophila melanogaster. Genetics 1975, 81:143-162.

82. Cagan RL, Ready DF: Notch is required for successive cell decisions in the developing Drosophila retina. Genes \& Dev 1989, 3:1099-III2.

Publish with BiolMed Central and every scientist can read your work free of charge

"BioMed Central will be the most significant development for disseminating the results of biomedical research in our lifetime. "

Sir Paul Nurse, Cancer Research UK

Your research papers will be:

- available free of charge to the entire biomedical community

- peer reviewed and published immediately upon acceptance

- cited in PubMed and archived on PubMed Central

- yours - you keep the copyright 